\title{
AVALIAÇÃO DAS TÉCNICAS DE PRECIPITAÇÃO QUÍMICA E ENCAPSULAMENTO NO TRATAMENTO E DESTINAÇÃO CONJUNTA DE RESÍDUOS LÍQUIDOS CONTENDO CROMO E VIDRARIAS DE LABORATÓRIO
}

\author{
Juliana Graciela Giovannini*, Glauco Arnold Tavares e José Albertino Bendassolli \\ Centro de Energia Nuclear na Agricultura, Universidade de São Paulo, CP 96, 13400-970 Piracicaba - SP, Brasil
}

Recebido em 14/3/07; aceito em 17/8/07; publicado na web em 10/3/08

\begin{abstract}
EVALUATION OF CHEMICAL PRECIPITATION AND ENCAPSULATION TECHNIQUES IN THE TREATMENT AND JOINT DISPOSAL OF LIQUID WASTES CONTAINING CHROMIUM AND LABORATORY GLASSWARE. In this paper, we present procedures for the treatment and final disposal of residual solutions containing chromium, in order to evaluate the effectiveness of chemical precipitation of the metal and the potential of the glass encapsulation technique, using broken laboratory glassware. The results demonstrated that $\mathrm{pH}$-values convenient for chemical precipitation are between 10 - 11. With regard to $\mathrm{Cr}(\mathrm{OH})_{3}$ encapsulation, the leaching and solubilization tests allowed to classify the waste as non-dangerous and non-inert. Finally, it is pointed out that the adoption of waste management practices in universities should be encouraged, helping to train professionals skilled in good laboratory practices.
\end{abstract}

Keywords: wastes; chromium; encapsulation.

\section{INTRODUÇÃO}

O gerenciamento dos resíduos sólidos industriais e domésticos é um dos principais problemas vivenciados nos dias atuais. Estimativas dão conta de que no estado de São Paulo são geradas anualmente mais de 500 mil t de resíduos Classe I (perigosos) e cerca de 20 milhões de $\mathrm{t}$ de resíduos Classe II A (não inertes e não perigo$\operatorname{sos}){ }^{1}$

Além das indústrias, as universidades e centros de pesquisa também acabam por gerar resíduos químicos, que, embora em pequenas quantidades, são encarados como um problema devido à diversidade com que são gerados, o que contribui para que essas instituições de ensino e pesquisa estejam, gradativamente, implementando seus Programas de Gerenciamento de Resíduos Quími$\cos ^{2}$

O primeiro trabalho desenvolvido no Centro de Energia Nuclear na Agricultura da Universidade de São Paulo (CENA/USP) que envolvia a gestão de resíduos químicos em um único laboratório (Laboratório de Isótopos Estáveis) data do 1998. Os principais resultados desta dissertação ${ }^{3}$ foram a proposição de algumas alternativas para o tratamento de resíduos e a caracterização da emissão de efluentes dos laboratórios.

Em 2001, contando com o fundamental apoio financeiro da Fundação de Amparo à Pesquisa do Estado de São Paulo (FAPESP), teve início o Programa de Gerenciamento de Resíduos Químicos e Águas Servidas (PGRQ) do CENA/USP. ${ }^{4}$ Os resíduos gerados no CENA/USP são bastante diversificados, incluindo solventes, soluções inorgânicas diversas $\left(\mathrm{NH}_{3} \mathrm{aq}, \mathrm{SO}_{2} \mathrm{aq}\right.$, ácidos, bases, soluções contendo metais, entre outras) e resíduos sólidos (óxido de cobre, perclorato de magnésio, etc). Nessa relação, destacam-se várias soluções residuais contendo cromo: soluções de sulfocrômica; soluções de dicromato de potássio; solução composta da mistura entre dicromato de potássio, ácido sulfúrico e sulfato ferroso amoniacal, geradas na determinação de biomassa microbiana em solos (Biomassa-C). ${ }^{5}$

\footnotetext{
*e-mail: jgiovann@cena.usp.br
}

Datam da segunda metade do século XX os primeiros registros de contaminação por metais pesados em água, entre os quais cromo e mercúrio. Desde aquela época, grande parte da responsabilidade pelos problemas ambientais verificados vem sendo atribuída ao desenfreado e acentuado desenvolvimento industrial desencadeado pela civilização humana. ${ }^{6}$

De forma a evitar que resíduos contendo esse metal sejam descartados no meio ambiente, algumas formas de tratamento foram desenvolvidas e relatadas. Na literatura, encontram-se várias técnicas de tratamento de cromo presente em resíduos líquidos, dentre as quais destacam-se a precipitação química, ${ }^{7}$ a retenção em resinas de troca iônica, ${ }^{8}$ a absorção em carvão ativado, ${ }^{9}$ a biossorção do cromo em cascas de arroz, ${ }^{10}$ a adsorção de cromo VI utilizando fibras de côco $^{11} \mathrm{e}$ vários outros, como redução eletroquímica, osmose reversa e extração por solventes. O grande número de técnicas citadas, longe de englobar todas as possibilidades, reforça a atual relevância dos procedimentos de tratamentos de resíduos.

Ao mesmo tempo, um outro resíduo freqüente dos laboratórios são restos de vidrarias originárias da quebra destes materiais. Ressalta-se que não há uma destinação especial para esses materiais, sendo comum o envio para reciclagem.

Recentemente, surgiram trabalhos que preconizam a imobilização de metais em material vítreo, o que possivelmente permitiria a destinação concomitante de ambos os resíduos (resíduos químicos e vidros). Segundo Delbianco Filho, ${ }^{12}$ a vitrificação é uma das melhores opções tecnológicas para imobilização de resíduos, visando a obtenção de um produto que não ofereça qualquer risco. A técnica consiste em agregar aos vidros e cerâmicas, resíduos sólidos (como o cromo) que possam conferir colorações a esses materiais. Assemelha-se, portanto, ao método de encapsulamento em matriz de cimento, bastante eficiente para metais pesados, como extensamente relatado na literatura. ${ }^{13}$

Diante do exposto, valendo-se das facilidades oferecidas nos laboratórios do CENA/USP e do Departamento de Física do Instituto de Geociências e Ciências Exatas da Universidade Estadual Paulista (IGCE/UNESP), investigou-se a eficiência das técnicas de precipitação química e encapsulamento com vistas a estabelecer uma possível destinação conjunta de resíduos contendo cromo e vidros gerados em atividades de ensino e pesquisa. 


\section{PARTE EXPERIMENTAL}

\section{Materiais}

Os equipamentos necessários ao desenvolvimento da pesquisa, além das vidrarias convencionais de laboratório (proveta, béquer, cápsulas de porcelana, bastões de vidro, entre outras), foram: medidor de $\mathrm{pH}$ modelo digital marca Orion modelo 4260-C15; capela exaustora Walk-in; agitador e aquecedor magnético (Marconi, mod 085); balança eletrônica digital modelo ER-182A, range $0.0001 \mathrm{~g}$, marca And; estufa ventilada; moinho de bola (Marconi, mod MR350); forno mufla, temperatura até $1300^{\circ} \mathrm{C}$; forno Maitec, mod. FET - 1600 vertical; cadinho de platina pura; forno Maitec, mod. FL - 1300/7; espectrômetro de absorção atômica (AAS), espectrômetro de emissão atômica com plasma acoplado indutivamente (ICPAES), metalizador MED 010 da Balzers, LinkOxford EXL acoplado ao microscópio eletrônico de varedura Zeiss DSM 940A.

Com relação aos reagentes e resíduos utilizados, destacam-se: ácido sulfúrico, hidróxido de sódio, tiossulfato de sódio, borato de sódio, ouro, solução de sulfocrômica residual, carbonato de sódio com prazo de validade vencido (resíduo sólido passivo) e vidrarias borossilicato (Pyrex).

\section{Tratamento químico}

Considerando-se as anteriormente citadas soluções residuais contendo cromo, geradas na instituição, optou-se pela realização dos ensaios iniciais utilizando-se alíquotas de $50 \mathrm{~mL}$ de uma solução residual de sulfocrômica com concentração de cromo total avaliada em 9,5 $\mathrm{g} \mathrm{L}^{-1}$. Vale ressaltar que o resíduo em questão foi devidamente segregado na fonte geradora e armazenado no entreposto de resíduos do CENA/USP em recipientes de polipropileno de $50 \mathrm{~L}$ de capacidade, em consonância com os procedimentos de gerenciamento de resíduos adotados nessa Instituição. ${ }^{4}$

$\mathrm{O}$ tratamento foi baseado na redução de $\mathrm{Cr}(\mathrm{VI})$ à $\mathrm{Cr}(\mathrm{III})$ em meio ácido, utilizando tiossulfato de sódio, conforme a Equação 1. A Equação 2 refere-se ao processo de precipitação química do cromo, realizado após a etapa de redução, estudando-se a elevação do $\mathrm{pH}$ da solução em valores variando entre 7 e 13. Após a precipitação, efetivada empregando-se solução de $\mathrm{NaOH} 6 \mathrm{~mol} \mathrm{~L}^{-1}$, o lodo formado passou por um processo de filtração e o volume filtrado foi neutralizado e encaminhado para determinação de cromo total através da técnica de espectrometria de absorção atômica, previamente ao descarte.

$$
\begin{aligned}
& 4 \mathrm{Cr}_{2} \mathrm{O}_{7}{ }^{2-}+3 \mathrm{~S}_{2} \mathrm{O}_{3}{ }^{2-}+26 \mathrm{H}^{+} \rightarrow 8 \mathrm{Cr}^{3+}+6 \mathrm{SO}_{4}{ }^{2-}+13 \mathrm{H}_{2} \mathrm{O} \\
& \mathrm{Cr}^{3+}+3(\mathrm{OH})^{-} \rightarrow \mathrm{Cr}(\mathrm{OH})_{3}
\end{aligned}
$$

Na seqüência, após determinar as condições ideais de precipitação, objetivando averiguar se a velocidade na adição da solução de hidróxido de sódio influenciaria na eficiência do processo, variou-se o tempo total de adição do precipitante $(0,5,10$ e $20 \mathrm{~min})$, sob agitação constante.

\section{Etapa de encapsulamento}

As vidrarias inutilizadas (resíduo classe II B, ABNT - NBR $10004)^{14}$ nos laboratórios são o principal insumo empregado na etapa de encapsulamento. Previamente a sua utilização, os materiais coletados junto aos laboratórios sofreram lavagens sucessivas com solução de ácido clorídrico $1 \mathrm{~mol} \mathrm{~L}^{-1}$ e água desionizada, seguidas de secagem e moagem. Ao vidro moído, juntou-se o resíduo de hidróxido de cromo (resíduo classe I, ABNT - NBR 10004) ${ }^{14}$ e o tetraborato de sódio (reagente auxiliar), em diferentes concentrações (Tabela 1). Essas misturas foram homogeneizadas e fundidas em forno mufla, utilizando cadinho de platina, à temperatura de $1450{ }^{\circ} \mathrm{C}$, durante cerca de $1 \mathrm{~h}$. Após isso, o material fundido foi vertido em moldes de aço e resfriado lentamente em forno pré-aquecido a $600{ }^{\circ} \mathrm{C}$.

Tabela 1. Composição das amostras preparadas para os testes de

\begin{tabular}{|c|c|}
\hline Testes & Composição \\
\hline $\mathrm{T} 1$ & $0,5 \% \mathrm{Cr}^{3+}+\mathrm{Na}_{2} \mathrm{~B}_{4} \mathrm{O}_{7}+$ vidro \\
\hline $\mathrm{T} 2$ & $0,5 \% \mathrm{Cr}^{3+}$ \\
\hline $\mathrm{T} 3$ & $+\mathrm{Na}_{2} \mathrm{CO}_{3}+$ vidro \\
\hline $\mathrm{T} 4$ & $1,5 \% \mathrm{Cr}^{3+} \quad\left({ }^{2}\right)^{3}$ \\
\hline T5 & $\mathrm{Na}_{2} \mathrm{~B}_{4} \mathrm{O}_{7}+$ vidro \\
\hline T6 & $\mathrm{Na}_{2} \mathrm{CO}_{3}+$ vidro \\
\hline
\end{tabular}
vitrificação

Para determinação da composição centesimal do material fundido, selecionaram-se aleatoriamente fragmentos $\left(0,1 \mathrm{~cm}^{2}\right)$ das amostras com superfície plana, os quais foram recobertos com película de ouro (10 a $20 \mathrm{~nm}$ ) empregando metalizador MED 010 da Balzers e levados para microanálise de raio $\mathrm{X}$ por espectrometria de energia dispersiva acoplada ao microscópio eletrônico de varredura.

\section{Ensaios para classificação dos resíduos}

Estes testes foram conduzidos seguindo o protocolo recomendado pela norma ABNT - NBR 10004 ${ }^{14}$ através dos ensaios de solubilização (ABNT - NBR 10005) ${ }^{15}$ e lixiviação (ABNT - NBR 10006), ${ }^{16}$ permitindo classificar os materiais vitrificados e também uma embalagem de bebida constituída em vidro com coloração esverdeada em função de suas periculosidades. Os testes estão descritos a seguir:

\section{Ensaios de solubilização}

A solubilização das amostras tamisadas em partículas inferiores a $9 \mathrm{~mm}$ foi avaliada através da extração com água desionizada, sob agitação, por $5 \mathrm{~min}$, seguida de período de descanso por 7 dias, após o qual, promoveu-se a filtração e a determinação do teor de cromo total no extrato solubilizado.

\section{Ensaios de lixiviação}

O ensaio de lixiviação difere do de solubilização no que se refere à solução extratora, representada por uma mistura de ácido acético e hidróxido de sódio em $\mathrm{pH}$ 4,93 ou solução de ácido acético em $\mathrm{pH}$ 2,88 , e também quanto ao período de agitação, que deve ser realizada a $30 \mathrm{rpm}$ por $18 \mathrm{~h}$.

\section{RESULTADOS E DISCUSSÃO}

\section{Tratamento por precipitação química}

A Figura 1 exibe as distintas eficiências de remoção do cromo observadas nos testes, realizados em triplicata, variando-se o $\mathrm{pH}$ entre 7 e 13. Ainda que, aparentemente, tenha sido confirmada uma significativa eficiência de remoção na faixa de $\mathrm{pH}$ entre 8 e 12, foi possível avaliar, através das análises realizadas no líquido sobrenadante, que o melhor intervalo de $\mathrm{pH}$ para precipitação química se situa entre 10 e 11 . É importante ressaltar que a realização de en- 
saios para se avaliar a melhor condição em que a precipitação do cromo ocorre se justifica em função da presença, na solução residual, de vários outros íons. Essas características particulares a cada tipo de resíduo explicam resultados experimentais que indicam que a condição ideal para uma melhor eficiência da reação possa, em algumas situações, ser um pouco diferente da publicada na literatura. ${ }^{17}$

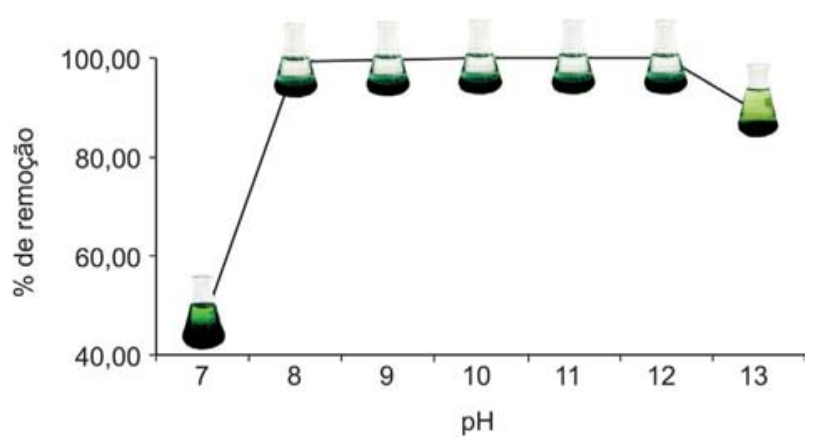

Figura 1. Influência da variação do pH na precipitação química do cromo

Em seguida, procederam-se os testes variando-se a velocidade de adição do hidróxido de sódio à solução residual até pH 10, sob agitação constante. Em todas as situações testadas, como mostra a Figura 2, as concentrações de $\mathrm{Cr}$ total determinadas no sobrenadante mantiveram-se em níveis próximos ao limite permitido para descarte, preconizado na Resolução CONAMA $357\left(0,5 \mathrm{mg} \mathrm{L}^{-1}\right) .{ }^{18}$ Assim sendo, sob o prisma da permissibilidade para descarte, parece ser indiferente adicionar lenta ou rapidamente a solução de $\mathrm{NaOH}$ durante o tratamento químico.

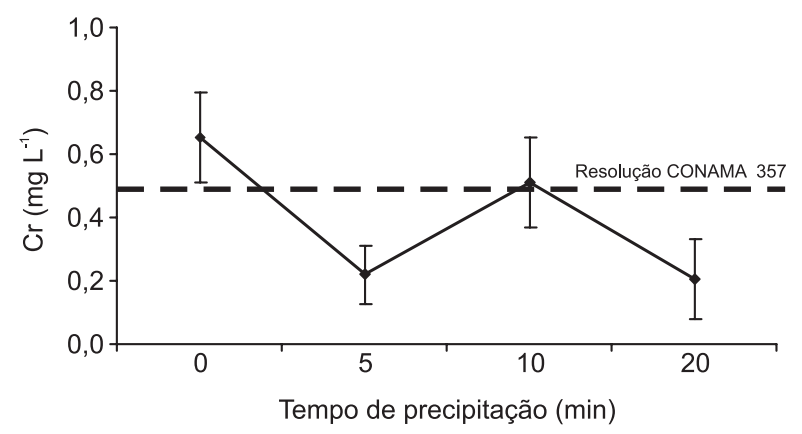

Figura 2. Teores de Cr total verificado no sobrenadante dos ensaios de precipitação variando-se o tempo de adição de hidróxido de sódio nas soluções residuais (linha pontilhada - resolução CONAMA 357)

\section{Testes de vitrificação}

Após a mistura e fusão de um resíduo classe I (hidróxido de cromo) com outro da classe IIB (vidrarias quebradas de laboratório) obteve-se um produto que foi preliminarmente denominado resíduo vitrificado contendo cromo. ${ }^{14} \mathrm{Na}$ Figura 3 podem ser vislumbradas as amostras dos materiais fundidos, vertidos em molde, com e sem adição de cromo.

Realizados os testes iniciais de vitrificação, verificou-se a dificuldade em amolecer o vidro borossilicato nas condições experimentais empregadas. Observando-se o diagrama ternário de fases publicado na literatura, ${ }^{19}$ permite-se compreender as temperaturas ideais para amolecimento do vidro em função das proporções de $\mathrm{Na}_{2} \mathrm{O}, \mathrm{B}_{2} \mathrm{O}_{3}$ e $\mathrm{SiO}_{2}$ constituintes desse material. De acordo com essse diagrama, a temperatura ideal para o amolecimento do vidro apro-

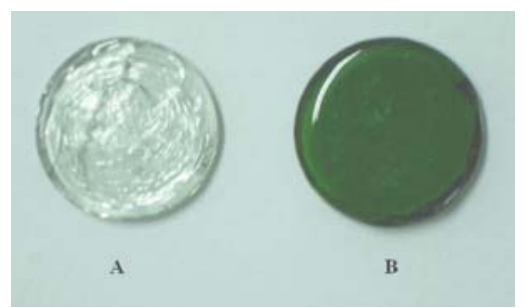

Figura 3. Amostras moldadas de vidro borossilicato fundido, sendo A amostra sem adição de cromo; e B-amostra contendo 0,5\% de cromo

xima-se a $600{ }^{\circ} \mathrm{C}$. Todavia, considerando-se a composição do vidro borossilicato, seria necessário que a fusão fosse realizada em temperatura superior a $1100{ }^{\circ} \mathrm{C}$, o que resulta em séria dificuldade operacional. Objetivando contornar esse problema, foi possível reduzir a temperatura do amolecimento do vidro para valores próximos a $600{ }^{\circ} \mathrm{C}$ através da adição de tetraborato de sódio (fundente), mantendo a estrutura do vidro praticamente inalterada. Durante este procedimento, a decomposição do fundente permitiu a liberação de pequenas quantidades de vapores de óxido de boro, que começaram a atacar as placas do forno compostas de cerâmica, o que é indesejável. Para evitar danos ao equipamento, propôs-se a substituição do tetraborato por carbonato de sódio, até então armazenado como resíduo sólido (prazo de validade expirado) no entreposto da Instituição, operacionalizando-se a fusão do vidro em temperatura aproximada a $700{ }^{\circ} \mathrm{C}$.

\section{Análise da composição do material vitrificado}

Quando se objetiva solidificar um resíduo em uma matriz qualquer, é conveniente o acompanhamento detalhado dessa operação, de forma a assegurar a efetividade do processo. Para isso, realizouse a determinação da composição centesimal dos componentes produzidos, comparando-se os resultados obtidos com a quantidade incorporada do cromo presente no resíduo. Os dados apresentados na Tabela 2 refletem os efeitos da adição de cromo e dos auxiliares de fusão na composição da matriz vítrea. Destacam-se nessa Tabela os resultados observados dos teores de $\mathrm{Cr}_{2} \mathrm{O}_{3}$ na matriz vítrea, que não refletiram com exatidão as concentrações adicionadas do metal, parecendo inclusive haver uma tendência de decréscimo nos teores de cromo incorporado ao material vítreo à medida que se aumenta a proporção da adição do metal. Entretanto, esse resultado deve ser interpretado com cautela, uma vez que, durante a fusão, não houve uma eficiente homogeneização dos reagentes, o que possibilitaria a distribuição desuniforme do metal. Com isso, considerando-se que para a realização das análises químicas utilizam-se pequenos fragmentos (cacos) do material fundido, os resultados poderiam não refletir a composição exata da peça produzida.

Tabela 2. Composição química do material vitrificado

\begin{tabular}{lccccc}
\hline Testes & $\begin{array}{c}\mathrm{SiO}_{2} \\
(\%)\end{array}$ & $\begin{array}{c}\mathrm{B}_{2} \mathrm{O}_{3} \\
(\%)\end{array}$ & $\begin{array}{c}\mathrm{Na}_{2} \mathrm{O} \\
(\%)\end{array}$ & $\begin{array}{c}\mathrm{Al}_{2} \mathrm{O}_{3} \\
(\%)\end{array}$ & $\begin{array}{c}\mathrm{Cr}_{2} \mathrm{O}_{3} \\
(\%)\end{array}$ \\
\hline $\mathrm{T} 1$ & 71,6 & 18,0 & 8,2 & 1,6 & 0,3 \\
$\mathrm{~T} 2$ & 73,6 & 10,1 & 14,0 & 2,0 & 0,6 \\
$\mathrm{~T} 3$ & 63,9 & 10,1 & 23,2 & 1,9 & 0,9 \\
$\mathrm{~T} 4$ & 65,0 & 10,1 & 18,8 & 1,5 & 1,2 \\
$\mathrm{~T} 5$ & 77,0 & 18,0 & 3,1 & 1,9 & - \\
T6 & 76,6 & 10,1 & 12,5 & 1,4 & - \\
\hline
\end{tabular}

Os teores dos demais componentes observados (Tabela 2) nas análises supracitadas refletem as composições aproximadas espe- 
radas em cada amostra, mesmo considerando-se a adição dos auxiliares de fusão.

\section{Ensaios para classificação de resíduos}

Na Tabela 3 são apresentados os resultados dos testes de lixiviação e solubilização realizados nas amostras de materiais vitrificados e na embalagem de bebida. De acordo com os testes de lixiviação realizados, todas as amostras puderam ser classificadas como resíduos não perigosos (classe II), uma vez que os resultados observados foram inferiores ao valor máximo permitido $\left(5 \mathrm{mg} \mathrm{L}^{-1}\right)$, preconizado no Anexo F da norma ABNT - NBR 10005. Ao mesmo tempo, os testes de solubilização demonstraram que praticamente não houve liberação de cromo pela embalagem de bebida analisada, o que permitiu classificá-la como resíduo não perigoso e inerte (classe II B), enquanto o material vitrificado permitiu a solubilização de cromo em valores acima do estabelecido pela norma, razão pela qual esse material deve ser classificado como resíduo não perigoso e não inerte (classe II A). A possível explicação para essa observação estaria atrelada às dificuldades técnicas no uso do vidro borossilicato para o processo de encapsulamento, uma vez que em trabalho publicado na literatura empregando vidro comum não foi relatada a solubilização do metal. ${ }^{12}$ Ressalta-se que, mesmo durante o preparo de amostras para a realização dos testes de lixiviação e solubilização, notou-se sensível diferença na etapa de moagem entre as amostras encapsuladas e o vidro da embalagem de bebida. Ocorre que, durante a trituração da embalagem de vidro, se formavam fragmentos muito mais uniformes e dúcteis, ao passo que as amostras de materiais encapsulados se fragmentavam mais facilmente.

Tabela 3. Resultados observados nos testes de lixiviação e solubilização

\begin{tabular}{lcc}
\hline Testes & $\begin{array}{c}\text { Lixiviação } \\
\left(\mathrm{mg} \mathrm{L}^{-1}\right)\end{array}$ & $\begin{array}{c}\text { Solubilização } \\
\left(\mathrm{mg} \mathrm{L}^{-1}\right)\end{array}$ \\
\hline T1 & $<0,02$ & $<0,02$ \\
T2 & 0,02 & $0,10^{*}$ \\
T3 & 0,04 & $4,53^{*}$ \\
T4 & 0,29 & $0,35^{*}$ \\
Embalagem de bebida & $<0,02$ & $<0,02$ \\
\hline
\end{tabular}

* Valor acima do limite máximo permitido no extrato (ABNT - NBR 10004).

\section{CONCLUSÕES}

A apreciação dos resultados obtidos permite concluir que o tratamento químico se mostrou eficiente e de prático manuseio, o que corrobora a sua utilização em rotina. Os aspectos ambientais e de segurança em química também reforçam essa aplicabilidade.

Quanto ao processo testado de encapsulamento, embora a técnica aparente ser promissora, no processo estudado, os resultados demonstraram o contrário. Todavia, esse resultado deve ser interpretado com cautela, uma vez que a solubilização do cromo do material vitrificado talvez não acontecesse caso um diferente tipo de vidro (por exemplo, vidro neutro) tivesse sido utilizado. Por fim, vale ressaltar que a realização de pesquisas dessa natureza é de grande interesse para a área de gestão de resíduos de laboratórios, contribuindo também para a disseminação de uma postura pró-ativa junto aos estudantes e profissionais.

\section{AGRADECIMENTOS}

À FAPESP pelo auxilio financeiro, ao Departamento de Física - IGCE/UNESP, Campus de Rio Claro, pela infra-estrutura concedida para a realização do trabalho e ao Núcleo de Apoio à Pesquisa em Microscopia Eletrônica Aplicada à Agricultura - ESALQ, onde foram realizadas análises de microscopia no vidro produzido.

\section{REFERÊNCIAS}

1. http://www.cetesb.sp.gov.br/Solo/residuos/industriais.asp, acessada em Março 2007.

2. Jardim, W. F.; Quim. Nova 1998, 21, 671; Cunha, C. J.; Quim. Nova 2001, 24, 424; Amaral, S. T.; Machado, P. F. L.; Peralba, M. C.; Camara, M. R.; Santos, T.; Berleze, A. L.; Falcão, H. L.; Martinelli, M.; Gonçalves, R. S.; Oliveira, E. R.; Brasil, J. L.; Araújo, . A.; Borges, A. C. A.; Quim. Nova 2001, 24, 419; Alberguini, L. B. A.; Silva, L. C.; Rezende, M. O. O.; Quim Nova 2003, 26, 291; Tavares, G. A. ; Bendassolli, J. A.; Quim. Nova 2005, 28, 732; Afonso, J. C.; Noronha, L. A.; Felipe, R. P.; Freidinger, N.; Quim. Nova 2003, 26, 602; Gerbase, A. E.; Gregório, J. R.; Calvete, T.; Quim. Nova 2006, 29, 397; Imbroisi, D.; Guaritá- Santos, A. J. M.; Barbosa, S. S.; Shintaku, S. F.; Monteiro, H. J.; Ponce, G. A. E.; Furtado, J. G.; Tinoco, C. J.; Mello, D. C.; Machado, P. F. L.; Quim. Nova 2006, 29, 404.

3. Tuono, V.; Dissertação de Mestrado, Universidade de São Paulo, Brasil, 1999.

4. Tavares, G. A.; Tese de Doutorado, Universidade de São Paulo, Brasil, 2004.

5. Vance, E. D.; Brookes, P. C.; Jenkinson, D. S.; Soil Biol. Biochem. 1987, 19, 703 .

6. Miller-Ihli, N. J. Em Hazardous metals in the environment; Stoeppler, M. ed.; Elsevier: Amsterdam, 1992, cap. 13; Bila, D. M.; Dezotti, M.; Quim. Nova 2007, 30, 651; Pimentel, L. C. F.; Chaves, C. R.; Freire, L. A. A. Afonso, J. C.; Quim. Nova 2006, 29, 1138; Ghiselli, G.; Jardim, W. F.; Quim. Nova 2007, 30, 695.

7. Lunn, G.; Sansone, E.B.; J. Chem. Educ. 1989, 66, 443.

8. Tenório, J. A. S. ; Espinosa, D. C. R.; Waste Management 2001, 21, 637.

9. Landrigan, R. B.; Hallowell, J. B.; Removal of chromium from plating rinse water using activated carbon, Washington: EPA, 1975, Report EPA 670/2$75-055$.

10. Guimarães, I. R.; Gorgulho, H. F.; Santos, J. M. S.; Martinelli, P. B.; Resumos da $28^{a}$ Reunião Anual da Sociedade Brasileira de Química, Poços de Caldas, Brasil, 2005.

11. Miranda, Jr. P.; Silva, P. R.; Suguiyama, S.; Máduar, M. F.; Resumos da $28^{a}$ Reunião Anual da Sociedade Brasileira de Química, Poços de Caldas, Brasil, 2005.

12. Delbianco Filho, S.; Dissertação de Mestrado, Universidade Estadual Paulista, Brasil, 2003.

13. Hanna, R. A.; Dissertação de Mestrado, Universidade de São Paulo, Brasil, 1990; Oliveira, K. D.; Dissertação de Mestrado, Universidade de São Paulo, Brasil, 1992; Chamie, S. L.; Dissertação de Mestrado, Universidade de São Paulo, Brasil, 1994; Cruz, R. A.; Hazardous residues disposure: chromium stabilization in cement, Engineering Information: Amsterdam, 1998.

14. ABNT - Associação Brasileira de Normas Técnicas; Classificação de resíduos sólidos, NBR - 10004, Rio de Janeiro, 2004.

15. ABNT - Associação Brasileira de Normas Técnicas; Lixiviação de resíduos, NBR -10005, Rio de Janeiro, 2004.

16. ABNT - Associação Brasileira de Normas Técnicas; Solubilização de resíduos, NBR -10006, Rio de Janeiro, 2004.

17. http://www.mtech.umd.edu/MTES/docs/PMG\%20metal\%20precip\%20man1.pdf, acessada em Maio 2007.

18. CONAMA; Resolução $n^{\circ}$ 357, Brasília: CONAMA, 2005.

19. Morey, G. W.; J. Soc. Glass Tech. 1951, 35, 270. 